\title{
Acute airway obstruction due to benign asymptomatic nodular goiter in the cervical region: A case report
}

\author{
TOKIKO ITO, KIYOSHI SHINGU, CHIKA MAEDA, MASATO KITAZAWA, YOSHIKI MIZUKAMI, \\ MANABU HIRAGURI, NAOTO HORIGOME, GENGO KANEKO, NOBUO ITOH and KEN-ICHI ITO
}

Division of Breast and Endocrine Surgery, Iida Municipal Hospital, Iida, Nagano 395-8502, Japan

Received June 10, 2014; Accepted March 2, 2015

DOI: $10.3892 / \mathrm{ol} .2015 .3464$

\begin{abstract}
Benign nodular goiter is a common disease. Although large goiters with obstructive symptoms including shortness of breath and dyspnea are a clear indication for surgery, acute upper airway obstruction, particularly in benign cervical goiter cases, is rare. We herein report the case of 46-year-old female with acute upper airway obstruction due to benign nodular goiter. The patient had a large and elastic goiter which was more pronounced on the left side of her neck, and surgery was scheduled for within a few months. Three months after the initial presentation, while still waiting for surgery, the patient was brought to the emergency room due to loss of consciousness and breathing difficulty and was immediately intubated. A computed tomography (CT) scan revealed that the trachea was markedly compressed by a nodular lesion in the left lobe, and bilateral pneumonia was also evident. Total thyroidectomy was immediately performed via the supraclavicular approach. The patient had an uneventful postoperative course and recovered well. The resected specimen included a well-encapsulated solid and cystic mass. Histopathological examination mainly revealed adenomatous goiter. The present case suggests that benign asymptomatic nodular goiter mostly located in the neck may cause acute airway obstruction, even if the nodules are not large. Early surgery should be performed when tracheal deviation and stenosis due to a large goiter is prominent by CT scan.
\end{abstract}

\section{Introduction}

Benign nodular goiter is a common disease affecting 5\% of the general population in non-endemic and $15 \%$ in endemic areas (1). The natural history of non-endemic benign goiters is characterized by a gradual increase in goiter size, with simultaneous development of increasing thyroid nodularity and thyroid

Correspondence to: Dr Kiyoshi Shingu, Division of Breast and Endocrine Surgery, Iida Municipal Hospital, 438 Yawata-machi, Iida, Nagano 395-8502, Japan

E-mail: 0374@imh.jp

Key words: adenomatous goiter, acute airway obstruction autonomy (2). There are four main treatment options for these diseases: monitoring without treatment, surgery, levothyroxine suppression and radioactive iodine-131 (3). Large goiters with obstructive symptoms including shortness of breath and dyspnea are a clear indication for surgery (4); however, acute upper airway obstruction, particularly in benign cervical goiter cases, is rare (5-9).

We herein report a rare case with acute upper airway obstruction due to a benign cervical nodular goiter.

\section{Case report}

A 46-year-old female was referred to Iida Municipal Hospital, Nagano, Japan, for treatment of a large goiter in December 2011. The patient had few complaints on presentation, but occasionally experienced difficulty breathing. She had a large, elastic and non-tender goiter which was more pronounced on the left side of her neck (Fig. 1A). Laboratory studies revealed a euthyroid state; antithyroglobulin and thyroid peroxidase antibodies were both negative. A computed tomography (CT) scan of the neck and thorax revealed gross enlargement of the left lobe of the thyroid, measuring $75 \times 51 \mathrm{~mm}$, with a clear border and a relatively homogeneous structure, which was mostly located in the cervical region (Fig. 1B). The trachea was narrowed and shifted to the right side by the nodular goiter. Fine needle aspiration biopsy revealed the nodule to be benign. The diagnosis of a benign nodular goiter was confirmed and surgery was scheduled for within a few months.

Three months later, the patient was brought to our emergency room by ambulance due to loss of consciousness with breathing difficulty and was immediately intubated for mechanical ventilation. The patient's height was $160 \mathrm{~cm}$ and she weighed $90 \mathrm{~kg}$. Carbon dioxide narcosis was indicated by arterial blood gas analysis: pH 6.76; pCO2, $106.5 \mathrm{mmHg}$; pO2, $46.3 \mathrm{mmHg}$. The CT scan revealed that the trachea was markedly compressed (the diameter of the narrowest portion of the lumen was $4 \mathrm{~mm}$ ) by the nodular lesion in the left lobe of the thyroid, and bilateral pneumonia was also evident (Fig. 1C and D). However, the size of the nodule itself was unchanged compared with that observed at the first visit. Total thyroidectomy was immediately performed via a supraclavicular approach without median sternotomy. We were able to fully palpate the nodule from the supraclavicular region to identify a well-encapsulated mass with a smooth surface. 

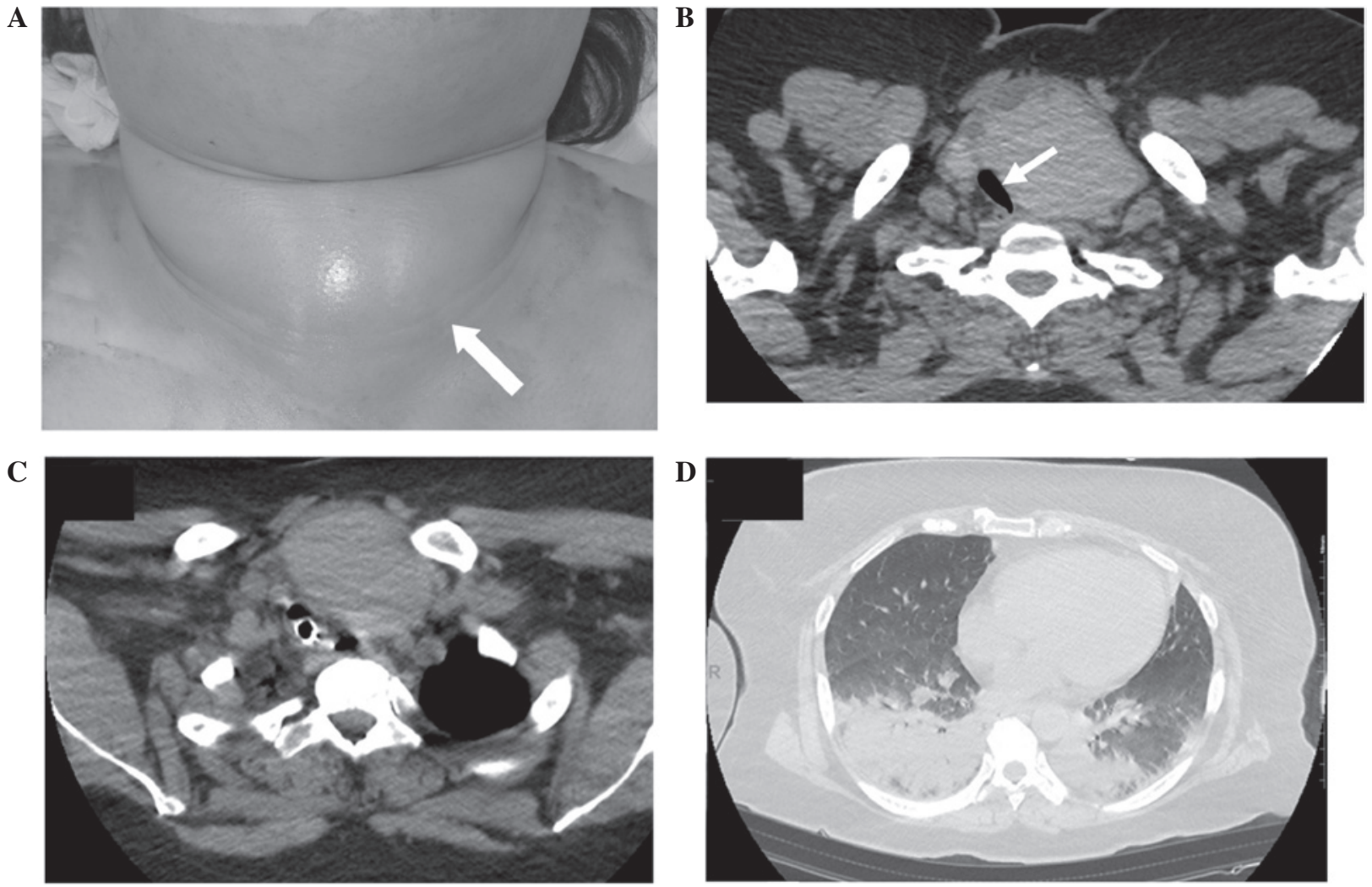

Figure 1. (A) Appearance of the neck of the patient. A large, elastic and non-tender goiter, which was more pronounced on the left side, was located in the neck (white arrowhead). (B) Computed tomography (CT) findings in the neck at the initial visit. The trachea was narrowed and shifted to the right side by the nodular goiter (white arrowhead). CT findings at the time of admission into the emergency room revealed that (C) the trachea was markedly compressed by the nodule, and (D) bilateral pneumonia was also evident.

Following removal of the thyroid, the trachea returned to the center of the neck and was normal with no features of tracheomalacia. The patient had an uneventful postoperative course and recovered well.

The resected specimen included a well-encapsulated solid mass measuring $45 \mathrm{~mm}$ in size. Part of the specimen was a monocular cyst. Histopathological examination mainly revealed an adenomatous goiter but there was a micro-papillary carcinoma in part of the goiter. The patient provided informed consent for publication of the present study.

\section{Discussion}

Acute airway obstruction due to retrosternal benign goiters or thyroid malignancies has been widely described, however, benign cervical goiters causing acute airway obstruction are rare (9). Deaths from such goiters due to, for example, suffocation by airway obstruction, should be prevented, considering that these are benign diseases which generally have a low mortality rate.

With respect to the risk factors for acute airway obstruction, studies have suggested that acute obstruction is due to sudden hemorrhage into a cyst, an upper respiratory tract infection causing edema, tracheal collapse or worsening of comorbidities $(5,8,10,11)$. Since the patient in our case was asymptomatic, it was decided not to operate immediately. It was not anticipated that such a nodular enlargement of the thyroid would cause sudden upper airway obstruction. There was no histological evidence of hemorrhage into the thyroid gland. The acute exacerbation could, however, have been due to upper airway infection or smoking, resulting in edema and retention of secretions, since the patient later caught a cold and, moreover, was a heavy smoker. Kadhim et al (12) reported that chronic obstructive airway disease, substernal extension and long-standing goiter were considered to be risk factors for developing acute, life-threatening airway compromise in the presence of benign thyroid disease. In addition, these authors advocated that following emergency thyroidectomy, close observation was mandatory to exclude airway compromise due to tracheomalacia and laryngeal edema. Nakadate et al (13) reported that the risk factors for tracheomalacia following thyroidectomy were as follows: goiter for more than 5 years, preoperative recurrent laryngeal nerve palsy, significant tracheal narrowing and/or deviation, retrosternal extension, difficulty with endotracheal intubation, reoperation and retrotracheal extension of a thyroid nodule. Fortunately, the present case did not have tracheomalacia, although the patient was carefully monitored for this condition following thyroidectomy in our intensive care unit.

Thus, it appears that the risk factors for acute benign cervical goiter causing acute airway obstruction are edema of the tracheal mucosa, secretion retention and bleeding. Edema of the tracheal mucosa is often caused by acute airway infection. Secretion retention is also caused by infection and certain other events, including chronic obstructive airway disease and smoking. Rapid enlargement of a nodular goiter due to bleeding from the cystic wall occasionally causes acute airway obstruction. 
In conclusion, it should be kept in mind that benign asymptomatic nodular goiter mostly located in the neck may cause acute airway obstruction, even if the nodules are not particularly large. Nodular goiters causing tracheal deviation and stenosis, visible on CT scans, necessitate that surgery be scheduled as soon as possible. If surgical treatment is delayed for any reason, patients should be strictly followed up until surgery, with attention paid to the above-mentioned risk factors.

\section{References}

1. Abraham D, Singh N, Lang B, et al: Benign nodular goitre presenting as acute airway obstruction. ANZ J Surg 77: 364-367, 2007.

2. Berghout A, Wiersinga WM, Smits NJ, et al: Interrelationships between age, thyroid volume, thyroid nodularity and thyroid function in patients with sporadic nontoxic goiter. Am J Med 89: 602-608, 1990.

3. Samuels MH: Evaluation and treatment of sporadic nontoxic goiter - some answers and more questions. J Clin Endocrinol Metab 86: 994-997, 2001

4. Clark OH, Duh QY and Kebebew E (eds): Textbook of Endocrine Surgery. 2nd edition. Saunders Elsevier, Philadelphia, 2005.
5. Sajja LR, Mannam GC, Sompalli S, et al: Multinodular goiter compressing the trachea following open heart surgery. Asian Cardiovasc Thorac Ann 14: 416-417, 2006.

6. Tseng KH, Felicetta JV, Rydstedt LL, et al: Acute airway obstruction due to a benign cervical goiter. Otolaryngol Head Neck Surg 97: 72-75, 1987.

7. Shaha AR: Surgery for benign thyroid disease causing trachea-oesophageal compression. Otolaryngol Clin North Am 23: 391-401, 1990

8. Melliere D, Saada F, Etienne G, et al: Goiter with severe respiratory compromise: evaluation and treatment. Surgery 103: 367-373, 1988.

9. Sharma A, Naraynsingh V and Teelucksingh S: Benign cervical multi-nodular goiter presenting with acute airway obstruction: a case report. J Med Case Rep 4: 258, 2010.

10. Rios A, Rodriguez JM, Galindo PJ, et al: Spirometric evaluation of respiratory involvement in asymptomatic multinodular goiter with an intrathoracic component. Arch Bronchoneumol 44: 504-506, 2008 (In Spanish).

11. Raftos JR and Etekk AT: Goitre causing acute respiratory arrest. ANZ J Surg 66: 331-332, 1996.

12. Kadhim AL, Sheahan P and Timon C: Management of life-threatening airway obstruction caused by benign thyroid disease. J Laryngol Otol 120: 1038-1041, 2006.

13. Nakadate Y, Fukuda T, Hara H, et al: Tracheomalacia after reoperation for an adenomatous goiter located in a unique position. J Anesth 25: 745-748, 2011. 